\title{
Imaging Carrier Diffusion in Perovskites with a Diffractive Optic- Based Transient Absorption Microscope
}

\author{
Zhenkun Guo, Ninghao Zhou, Olivia F. Williams, Jun Hu, Wei You, ${ }^{\circledR}$ and Andrew M. Moran* \\ Department of Chemistry, University of North Carolina at Chapel Hill, Chapel Hill, North Carolina 27599, United States
}

ABSTRACT: Carrier diffusion is imaged in a perovskite film and crystal using a newly developed transient absorption microscope. Wide-field imaging is combined with a diffractive optic-based beam geometry to conduct 41 transient absorption experiments in parallel in this experimental setup. This configuration allows statistics to be quickly compiled with a $1 \mathrm{kHz}$ laser system. Diffusion coefficients of 0.01 and $0.20 \mathrm{~cm}^{2} / \mathrm{s}$ are obtained for the methylammonium lead iodide film and crystal, respectively. Our data suggest that the dynamics in the film are dominated by intensity dependence of the carrier lifetimes as opposed to carrier diffusion. The small diffusion coefficients determined in the film are attributed to the presence of grain boundaries.

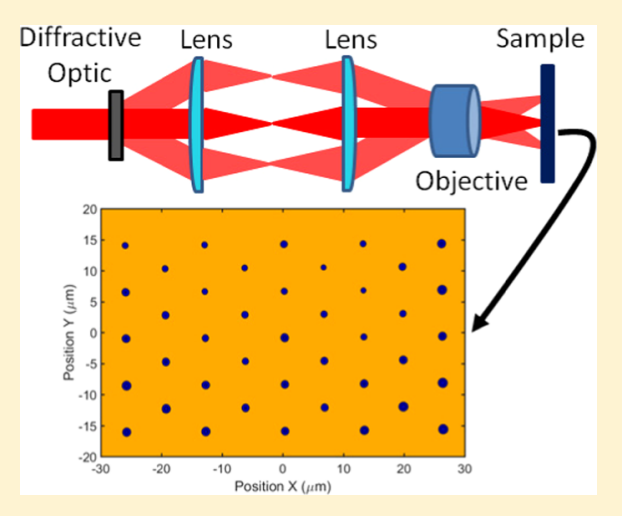

\section{INTRODUCTION}

Organohalide perovskites carry many advantages for use in solar cells and other optoelectronic devices. ${ }^{1-3}$ They can be processed from solution ${ }^{4}$ and have absorbance spectra that are readily tuned with control of the halide ratio ${ }^{5}$ and metal cation. ${ }^{6}$ Long diffusion lengths ${ }^{7,8}$ and low susceptibility to trap-assisted recombination ${ }^{9,10}$ have also been demonstrated, which is a remarkable result considering the flexibility of the ionic lattice. ${ }^{11}$ Slow vibrational cooling, which occurs on the time scale of $100 \mathrm{ps}$, is one source of enhanced carrier transport in perovskites. ${ }^{12-15}$ The extent to which grain boundaries and many-body decay processes influence carrier transport is also under investigation. $8,16,17$

Carrier mobilities in organohalide perovskites have been determined using a variety of methods. Conventional (indirect) measurements of carrier diffusivities yield values (0.02-0.16 $\left.\mathrm{cm}^{2} / \mathrm{s}\right)^{7,14,18}$ that are an order of magnitude smaller than those obtained in a more direct manner with transient absorption microscopy $\left(0.05-1.77 \mathrm{~cm}^{2} / \mathrm{s}\right) .^{15,19-21}$ Discrepancies in the diffusion coefficients determined using these two approaches are thought to represent the influence of grain boundaries. ${ }^{21}$ In the conventional approach, an electron ([6,6]-phenyl- $\mathrm{C}_{61}{ }^{-}$ butyric acid methyl ester) or hole quencher (Spiro-OMeTAD) is placed in contact with the perovskite. Diffusion coefficients can then be determined under the assumption that electron transfer is instantaneous when an electron reaches the interface with the quencher. Although the conventional approach is often effective, the rate of electron transfer and the quenching efficiency can vary significantly at different locations on heterogeneous interfaces. ${ }^{22}$ Such a breakdown in assumptions may lead to an underestimation of diffusion coefficients. By contrast, carrier diffusion within individual grains can be directly imaged with transient absorption microscopy, thereby eliminating contributions from grain boundaries when the laser is focused to a spot size smaller than an individual grain.

Ultrafast transient absorption microscopy is a relatively new approach for studying carrier diffusion in nanoparticles and films. ${ }^{15,19-21,23-27}$ Systems under investigation include silicon nanowires, $^{24}$ metal dichalcogenides, ${ }^{26}$ perovskite thin films, ${ }^{15,19,21}$ and various organic semiconductors. ${ }^{23}$ In these types of experiments, the pump beam is typically focused to a sub-micrometer spot size and a probe beam is raster scanned to image carrier diffusion. Laser systems with megahertz repetition rates are best-suited to such point-by-point methods for image construction. In an alternate approach that is more appropriate for laser systems with kilohertz repetition rates, an entire femtosecond time-resolved image is obtained in a wide-field geometry without scanning the position of a laser beam. ${ }^{28,29}$ Unfortunately, wide-field imaging is not well-suited for studies of carrier diffusion because the pump and probe beams are focused to large spot sizes on the sample. A method that combines wide-field imaging with the initiation of a localized distribution of carriers (on the micrometer length scale) is better-suited for laser systems with kilohertz repetition rates.

In this work, we describe a diffractive optic-based, wide-field method for conducting transient absorption microscopy. To directly image carrier diffusion in organohalide perovskite systems, the pump and probe beams, which counter propagate through the microscope objective, and are focused to 0.7 and $150 \mu \mathrm{m}$ spot sizes on the sample surface, respectively. Up to 41 transient absorption experiments are conducted in parallel using an array of pump laser beams generated with a diffractive optic. This multibeam approach facilitates the rapid attainment

Received: April 17, 2018

Published: April 30, 2018 
of statistical information. The capabilities of the instrument are demonstrated with measurements conducted on lead iodide perovskite films and single crystals. This choice of systems is motivated by two goals. The first goal is to analyze the effects of grain boundaries on the diffusivities and relaxation processes. The second goal is to establish a fitting procedure that accounts for the dependence of the excited state lifetime on the positiondependent laser intensity.

\section{EXPERIMENTAL METHODS}

II.I. Sample Preparation. Methylammonium lead iodide perovskite films were prepared based on previously reported methods. ${ }^{30}$ Dimethylformamide (DMF) was obtained from Sigma-Aldrich, lead iodide was purchased from Alfa Aesar, methylammonium iodide (MAI) was obtained from Solaronix, and isopropanol (Optima grade) was purchased from Fisher Scientific. All materials were used without further purification.

Fluorine-doped tin oxide (FTO) (Sigma-Aldrich; $\sim 13 \Omega /$ sq, approx. $1.1 \mathrm{~cm}^{2}$ ) substrates were cleaned via sonication for 20 min in a series of four solvents: Contrex $\mathrm{AP}$ glassware detergent in deionized water, deionized water, acetone, and isopropanol. Substrates were dried in a stream of nitrogen gas following the isopropanol wash.

Precleaned FTO substrates and a lead iodide solution $(0.83$ $\mathrm{M}$ in DMF) were preheated at $90{ }^{\circ} \mathrm{C}$ for $30 \mathrm{~min}$ immediately before film deposition. Perovskites films were prepared using a two-step fabrication process. First, $50 \mu \mathrm{L}$ of the preheated lead iodide solution was deposited onto the preheated substrate by spin-coating at $6000 \mathrm{RPM}$ for $20 \mathrm{~s}$ (3000 RPM/s acceleration). Immediately following the spin-coating, $200 \mu \mathrm{L}$ of MAI solution $(10 \mathrm{mg} / \mathrm{mL}$ in isopropanol) was deposited on top of the lead iodide layer and allowed to soak into the film for $10 \mathrm{~s}$. Excess solvent was spun off the film using the previously described spin cycle. The perovskite films were then annealed at $90{ }^{\circ} \mathrm{C}$ for $40 \mathrm{~min}$.

The perovskite crystal was prepared with the space-limiting method reported elsewhere. ${ }^{31}$ First, $461 \mathrm{mg}$ of lead iodide and $159 \mathrm{mg}$ of MAI were dissolved in $0.75 \mathrm{~mL} \gamma$-butyrolactone (from Sigma-Aldrich) and heated at $60{ }^{\circ} \mathrm{C}$. A drop of the precursor solution was then deposited onto a glass slide (Fisher Scientific, precleaned and used as received) and covered with a second identical slide. Thin perovskite crystals were then grown between the glass slides at $120{ }^{\circ} \mathrm{C}$ for $2 \mathrm{~h}$.

II.II. Transient Absorption Microscopy. All experiments are conducted with a $45 \mathrm{fs}, 4 \mathrm{~mJ}$ Coherent Libra laser system with a $1 \mathrm{kHz}$ repetition rate. Continuum pump and probe pulses are produced by focusing $1.5 \mathrm{~mJ}$ of the $800 \mathrm{~nm}$ fundamental beam into a $2 \mathrm{~m}$ long tube filled with argon gas to induce continuum generation. The continuum is then divided into pump and the probe beams with a 70:30 beam splitter. The two laser beams are passed through all-reflective 4F setups, which are based on $1200 \mathrm{~g} / \mathrm{mm}$ gratings and $20 \mathrm{~cm}$ focal length mirrors. Motorized slits at the Fourier planes are used to filter the desired portions of the pump and probe spectra. The spectrally filtered pulses have $250 \mathrm{fs}$ durations and $5 \mathrm{~nm}$ widths. The continuum beams are then relayed into the microscope setup, shown in Figure 1c. Pulse energies are controlled with neutral density filters.

The operations performed by the diffractive optic-based microscope are illustrated in Figure 1a. Three beams are shown for clarity; however, arbitrary beam patterns can be generated by customizing the diffractive optic. ${ }^{32,33}$ The pump is split into 41 beams with the diffractive optic (Holoeye) employed in this

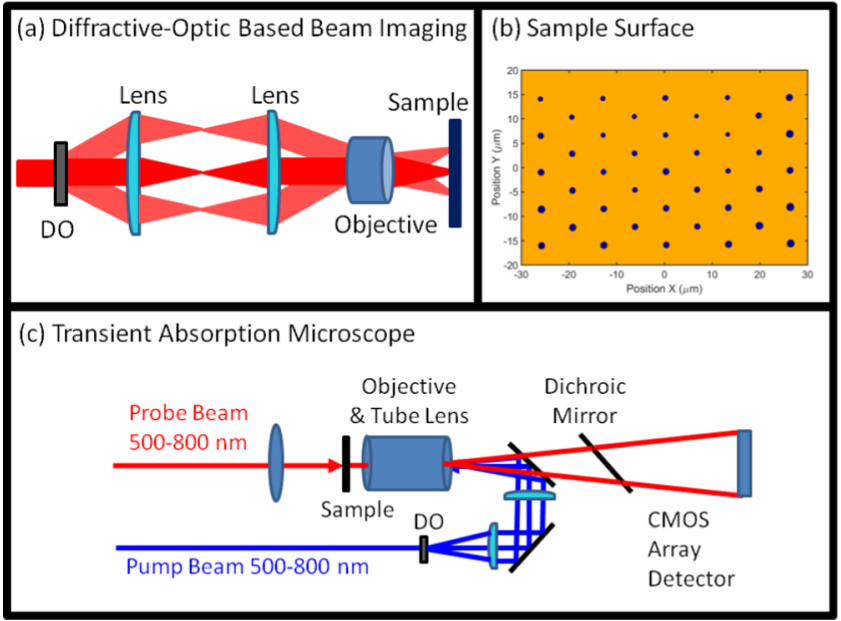

Figure 1. Transient absorption microscopy experiments are conducted with a diffractive optic-based, wide-field microscope. (a) A diffractive optic is used to generate an array of pump beams which focus at different places on the sample. (b) Array of 41 pump laser beams is focused onto the sample surface. (c) Counter-propagating pump and probe beams are focused to full width at half-maximum (FWHM) spot sizes of 0.7 and $150 \mu \mathrm{m}$, respectively.

work (see Figure $1 \mathrm{~b}$ ). The two lenses placed before the microscope objective have 10 and $2.5 \mathrm{~cm}$ focal lengths, which reduce the angles between beams by $75 \%$. The pump beams are then focused onto the sample with a microscope objective (Olympus). The infinity-corrected microscope objective has a magnification of $40 \times$, a numerical aperture of 0.95 , and a working distance of $0.18 \mathrm{~mm}$. The tube lens located between the objective and the detector is also infinity corrected and has an $18 \mathrm{~cm}$ focal length. With this objective, the FWHM spot size of each pump beam is $0.7 \mu \mathrm{m}$ on the sample surface. The probe beam, which has a fluence of approximately $15 \mu \mathrm{J} / \mathrm{cm}^{2}$ at the sample, is focused to a spot size of $150 \mu \mathrm{m}$ from the opposite side of the microscope objective using a $10 \mathrm{~cm}$ focal length lens. Therefore, the probe intensity for a spot at one of the corners of the array of pump beams is $11 \%$ less than that of the central beam (see Figure 1b). Of course, a difference of $11 \%$ is irrelevant in transient absorption experiments because differential absorption is independent of the probe intensity.

The complementary metal-oxide-semiconductor detector is 12-bit with a $1024 \times 1280$ array of pixels and $915 \mathrm{~Hz}$ maximum frame rate (Phantom Miro C110). The detector is synchronized to the laser system at $1 \mathrm{kHz}$. An optical chopper, which is placed in the path of the pump beam, cycles the instrument between the pump-on, $S_{1}$, and pump-off, $S_{2}$, conditions at $500 \mathrm{~Hz}$. Differential absorption is then computed as $\Delta A=\log \left(S_{1}\right)-\log \left(S_{2}\right)$. Each differential absorption image is averaged over a total of 100 images in a single scan of the delay line. The delay line is scanned 30 times during each experiment. The signal profiles are overlaid for scans $1-30$ to rule out sample degradation. We do not observe variation in the signal profiles for data acquisition times up to $90 \mathrm{~min}$.

\section{SIGNAL PROCESSING}

Sensitivity of the photoluminescence quantum yield to the incident light intensity has been demonstrated in previous studies of organohalide perovskites. ${ }^{34-37}$ The quantum yield of light emission is compared to the spatially averaged intensity (i.e., averaged over the beam profile) of the incident laser beam 

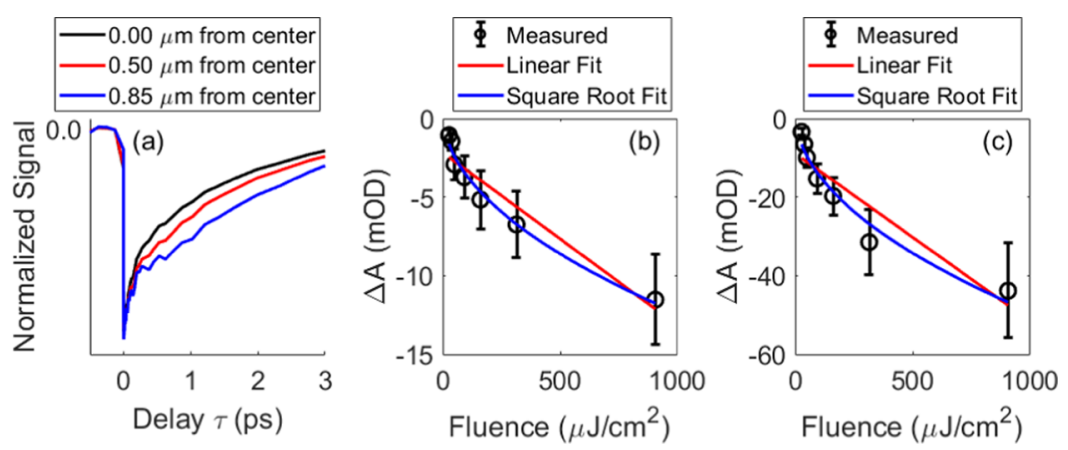

Figure 2. Control experiments are conducted to establish a model for signal processing. (a) The decay rate decreases with distance from the center of one of the pump beams because of the decrease in laser fluence. Transient absorption signals measured at $760 \mathrm{~nm}$ for the (b) film and (c) crystal exhibit square root dependencies on the fluence of the pump beam (pump is at $570 \mathrm{~nm}$ ).

in these measurements. In the present experiments, it is important to account for variation of the carrier lifetimes across the Gaussian beam profile. As expected, we find that the lifetime is shortest in the center of the pump beam and increases at points displaced from the peak of the intensity distribution (see Figure 2a). Such variation of the lifetime promotes a delay-dependent expansion in the width of the signal spot that is unrelated to carrier diffusion. Incorporating this process into the algorithm for signal interpretation is important because the carrier diffusivity will be overestimated if the intensity dependence of the lifetime is not taken into account.

The relaxation dynamics under investigation exhibit a clear dependence on the pump intensity, as evidenced by variation of the lifetime within the laser spot. We account for such manybody relaxation processes with the modified diffusion equation

$$
\frac{\partial N}{\partial \tau}=D_{0} \frac{\partial^{2} N}{\partial x^{2}}-\frac{N}{\tau_{1}}-\frac{N^{2}}{\tau_{2}}
$$

where $x$ is a spatial coordinate (a one-dimensional slice through the peak of a two-dimensional Gaussian intensity distribution), $N$ is the carrier density, $D_{0}$ is a diffusion constant, $\tau_{1}$ is the single-body carrier lifetime, and $\tau_{2}$ is a two-body relaxation time. The carrier density, $N$, is linear in the fluence of the pump beam, which is a directly measured quantity. The proportionality factors estimated for the film and crystal are $4.23 \times 10^{18}$ and $4.23 \times 10^{18} \mathrm{~cm}^{-1} \mu \mathrm{J}^{-1}$, respectively. For example, the carrier densities calculated for the film and crystal are $4.23 \times$ $10^{18}$ and $4.52 \times 10^{18} \mathrm{~cm}^{-3}$, respectively, at $27 \mu \mathrm{J} / \mathrm{cm}^{2}$. The model used to estimate carrier densities is described in the Supporting Information.

Data processing involves calculating full-width-half-maxima of the signal spots at each delay point. The signal intensity is first normalized to 1 for each one-dimensional slice of the signal. On the left (smaller $x$ value) side of the peak, two successive data points $\left(x_{1}, y_{1}\right),\left(x_{2}, y_{2}\right)$ are located at a signal intensity of 0.5 . The half-width-half-maximum (HWHM) coordinate on this side of the peak is then calculated with linear interpolation

$$
\mathrm{HWHM}_{\text {left }}=\left(0.5-y_{1}\right) \cdot \frac{x_{2}-x_{1}}{y_{2}-y_{1}}+x_{1}
$$

The relation between the signal intensity $S(x, \tau)$ and carrier density $N(x, \tau)$ must be known to fit the data. We have confirmed the signal scales as the square root of $N(x, \tau)$

$$
S(x, \tau)=A \sqrt{N(x, \tau)}
$$

where $A$ is a linear scaling parameter determined in the fitting process (see Figure $2 \mathrm{~b}, \mathrm{c}$ ). Such scaling is anticipated when interactions between electronic excitations are significant. ${ }^{13,15,38}$ The initial condition required to solve eq 1 is given by

$$
N(x, 0)=\left(\frac{S(x, 0)}{A}\right)^{2}
$$

The boundary condition for the differential equation is

$$
N( \pm \infty, 0)=0
$$

The least squares method is used to fit the data and obtain the parameters $D_{0}, A, \tau_{1}$, and $\tau_{2}$.

\section{RESULTS AND DISCUSSION}

Both the film and crystal exhibit intense stimulated emission resonances near $760 \mathrm{~nm}$, as shown in Figure 3. Evidence for excitons has been presented for perovskites with crystalline domains in recent literature; ${ }^{27,39}$ however, we do not observe signatures of excitons in this crystal (i.e., no excited state absorption in the $700-800 \mathrm{~nm}$ range). The transient absorption spectrum of the film red shifts at delay times less than $10 \mathrm{ps}$, whereas the peak of the signal is essentially independent of the delay time for the crystal. This difference between the spectra is partly due to a larger excited state absorption response in the film at detection wavelengths less than $740 \mathrm{~nm}$. To compare both systems on similar footing, images are constructed with signal detection at $760 \mathrm{~nm}$. The dynamics of interest occur on the nanosecond time scale and are therefore insensitive to the ultrafast red shift in the signal spectrum of the film.

Transient absorption images measured for the perovskite film and crystal are presented in Figure 4. In both systems, the signal spots expand as the delay time increases due to carrier diffusion and/or intensity dependence of the carrier lifetimes. These two processes cannot be distinguished by inspection. Rather, it is necessary to vary the intensities of the pump beams and fit the intensity distributions of the signals. In addition, the shape of the signal intensity is maintained as time evolves for carrier diffusion, whereas the peak of the signal "flattens" when intensity dependence of the excited state lifetimes takes hold. Although eq 1 is sufficient for such line shape analysis, conclusions about the influence of the pump intensity on the widths of the signal spots should be corroborated with 

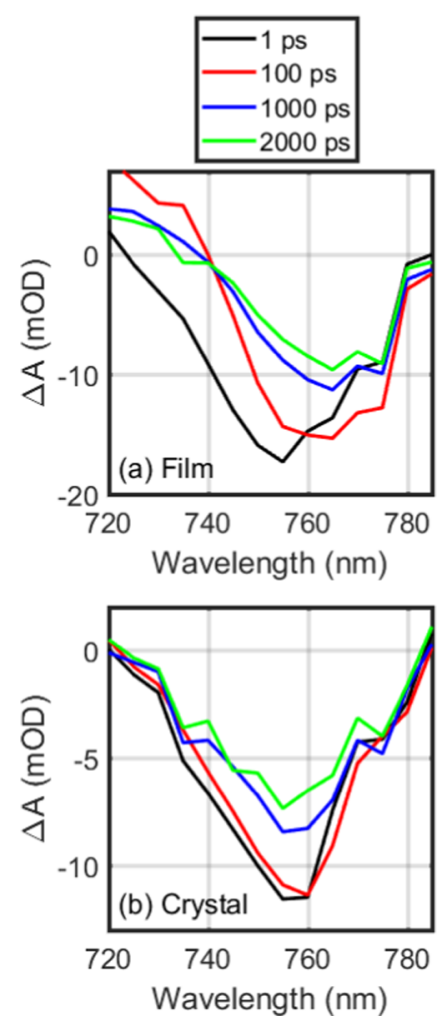

Figure 3. Transient absorption spectra measured for the perovskite (a) film and (b) crystal with a $570 \mathrm{~nm}$ pump wavelength. Imaging for both systems is conducted at probe wavelength of $760 \mathrm{~nm}$.

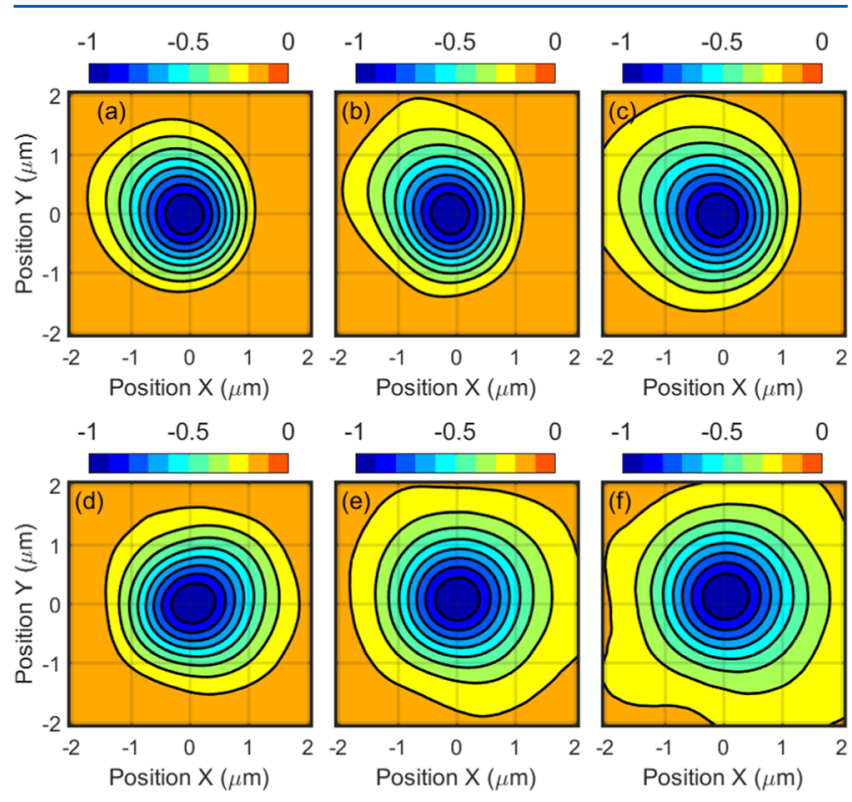

Figure 4. Transient absorption signals measured with $570 \mathrm{~nm}$ pump beam and $760 \mathrm{~nm}$ probe beam with a pump fluence of $40 \mu \mathrm{J} / \mathrm{cm}^{2}$. Images measured for a perovskite film at delay times of (a) $1 \mathrm{ps,} \mathrm{(b)}$ $500 \mathrm{ps}$, and (c) $1000 \mathrm{ps}$. Images measured for a perovskite single crystal at delay times of (d) 1 ps, (e) 500 ps, and (f) 1000 ps. The sizes of the signal spots expand as the delay time increases because of carrier diffusion and intensity dependence of the carrier lifetimes.

measurements, in which the intensity of the pump is varied over a wide range.

Dynamics in the spatial widths of the transient absorption signal spots are displayed in Figure 5. Equation 1 adequately describes the dynamics in the spot widths for both samples at all pump fluences. In the film, the magnitude of the expansion in the spot widths becomes larger as the pump fluence increases, whereas the spot widths are fairly insensitive to the pump fluence in the crystal. The behavior of the film suggests that the spot widths increase primarily because the carrier lifetimes are shortest in the center of the signal spot (see Figure $2 a$ ). This fluence-dependent effect on the spot widths is associated with the two-body contribution to the relaxation time, $\tau_{2}$. In contrast, the delay-dependent increases in the spot widths determined for the crystal are insensitive to the fluence of the pump, which indicates that carrier diffusion is responsible for the dynamics.

The fitting parameters obtained for the perovskite film and crystal are compared in Figure 6. The error bars span ranges equal to 2 standard deviations. The parameters are obtained by fitting the transient absorption dynamics at each spot on the sample surface with eq 1 . The means and standard deviations for each of the parameters are then calculated for 41 and 33 spots for the film and crystal, respectively. Fewer laser spots are processed for the crystal because of light scattering at defects.

The diffusion coefficients, $D_{0}$, determined for the single crystal are near $0.20 \mathrm{~cm}^{2} / \mathrm{s}$ for most pump fluences, whereas those obtained for the film are close to $0.01 \mathrm{~cm}^{2} / \mathrm{s}$. Diffusivities of the carriers in the film are below the lower limit of the range of values reported in earlier studies $\left(0.02-0.16 \mathrm{~cm}^{2} / \mathrm{s}\right){ }^{7,14,18}$ In contrast, the diffusion coefficients determined for the crystal are within the range of those reported in recent transient absorption microscopy measurements conducted on individual grains $\left(0.05-1.77 \mathrm{~cm}^{2} / \mathrm{s}\right) \cdot{ }^{15,19-21}$ The smaller diffusivities determined for carriers in the film are consistent with the dynamics in the spot widths displayed in Figure 5. Notably, the parameters, $D_{0}$ and $\tau_{2}$, are governed by dynamics in the shapes of the signal spots (i.e., the peak of the signal spot flattens when two-body dynamics contribute). For this reason, Figures 5 and 6 represent independent ways to differentiate diffusion and intensity dependence of the carrier lifetimes.

Our data suggests that the difference in diffusion coefficients, $D_{0}$, obtained for the film and crystal is associated with grain boundaries. The grain sizes in the film are on the order of 0.2 $\mu \mathrm{m}$, which is smaller than $0.7 \mu \mathrm{m}$ spot sizes of the pump laser. Thus, the experimental measurements are sensitive to intergrain carrier diffusion processes in the film (i.e., insensitive to diffusion within the grains). This may explain why the diffusion constants for the film derived from our measurements are smaller than those reported in recent transient absorption microscopies. ${ }^{15,19,21}$

\section{CONCLUSIONS}

In summary, the present approach for conducting transient absorption microscopy solves several technical challenges associated with the use of a $1 \mathrm{kHz}$ laser system. First, the data acquisition time is minimized by employing a wide-field geometry, in which the pump and probe beams counter propagate through the sample. The pump beams are focused to spot sizes with FWHM of $0.7 \mu \mathrm{m}$, whereas the spot size of the probe beam is $150 \mu \mathrm{m}$. In this configuration, an entire image of the sample can be obtained in less than $1 \mathrm{~s}$ without raster scanning individual laser beams on the sample surface. Second, a diffractive optic is used to generate an array of 41 pump beams. Conducting many transient absorption experiments in parallel allows statistics to be quickly compiled. 


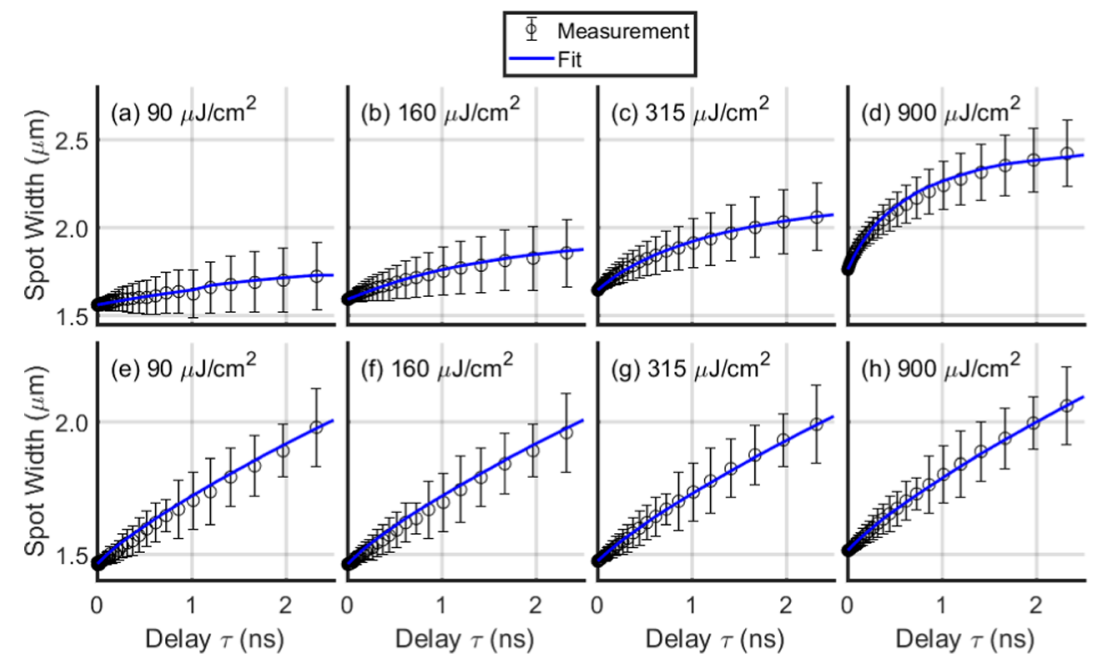

Figure 5. Full-width-half-maximum spot widths measured for a methylammonium lead iodide perovskite film (top row) and single crystal (bottom row). The error bars represent 2 standard deviations, which are calculated for 41 and 33 spots on the film and crystal, respectively. These data suggest that dynamics in the film and crystal are associated with fluence-dependent carrier lifetimes and carrier diffusion, respectively.

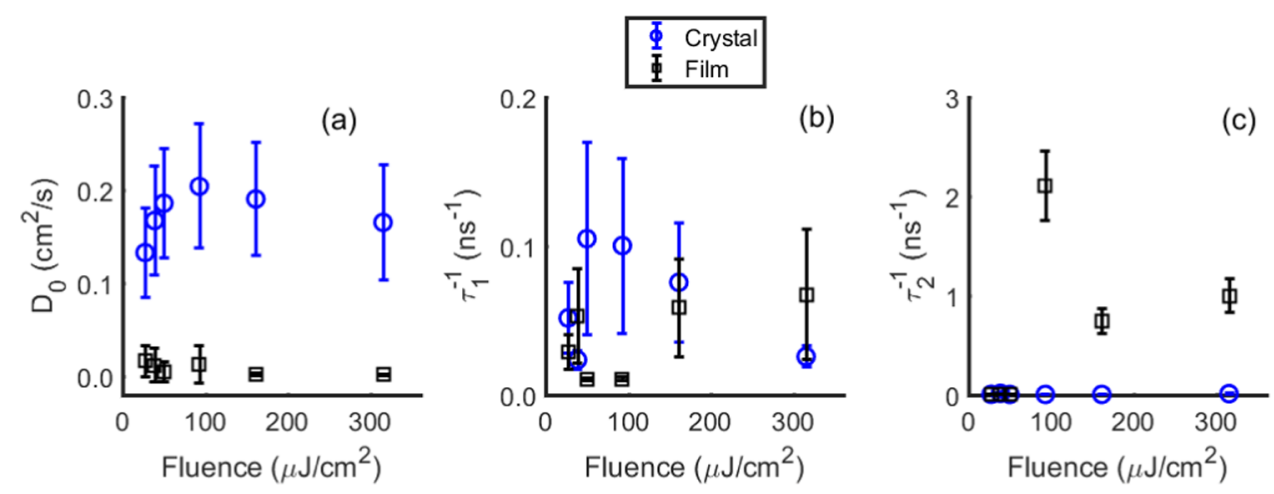

Figure 6. Statistical analyses of fitting parameters for perovskite film and single crystal: (a) diffusion coefficients, $D_{0}$; (b) single-body relaxation rate, $\tau_{1}^{-1} ;$ (c) two-body relaxation rate, $\tau_{2}^{-1}$. The uncertainty intervals represent 2 standard deviations. These data confirm the negligibility of carrier diffusion in the perovskite film. In contrast, expansion of the signal spots observed for the perovskite crystal is dominated by carrier diffusion.

The capabilities of the instrument are demonstrated with applications to a methylammonium lead iodide perovskite film and crystal. Our data suggests that carrier diffusion in the film is essentially negligible on the nanosecond time scale, whereas the diffusion coefficients in the crystal are approximately $0.2 \mathrm{~cm}^{2} / \mathrm{s}$. Measurements conducted at multiple pulse energies are required to distinguish between two mechanisms for delaydependent expansions in the spot widths: carrier diffusion and intensity dependence of the carrier lifetimes. We conclude that the spot widths expand in the film because of intensity dependence of the carrier lifetimes rather than carrier diffusion. These differences between the film and crystal are attributed to the presence of grain boundaries in the film.

\section{ASSOCIATED CONTENT}

\section{S Supporting Information}

The Supporting Information is available free of charge on the ACS Publications website at DOI: 10.1021/acs.jpcc.8b03643.

Scanning electron microscope images are presented for the film and crystal; the model used to estimate carrier densities is described; fitting parameters are plotted for each pump spot over a range of fluences for both the film and crystal; all fitting parameters are tabulated (PDF)

\section{AUTHOR INFORMATION}

\section{Corresponding Author}

*E-mail: ammoran@email.unc.edu.

ORCID $\odot$

Wei You: 0000-0003-0354-1948

Notes

The authors declare no competing financial interest.

\section{ACKNOWLEDGMENTS}

This work is supported by the National Science Foundation under CHE-1504350 (Z.G. and A.M.M.) and DMR-1610879 (J.H. and W.Y.). SEM images were collected at the Chapel Hill Analytical and Nanofabrication Laboratory, CHANL, a member of the North Carolina Research Triangle Nanotechnology Network, RTNN, which is supported by the National Science Foundation, Grant ECCS-1542015, as part of the National Nanotechnology Coordinated Infrastructure, NNCI.

\section{REFERENCES}

(1) Lee, M. M.; Teuscher, J.; Miyasaka, T.; Murakami, T. N.; Snaith, H. J. Efficient Hybrid Solar Cells Based on Meso-Superstructured Organometal Halide Perovskites. Science 2012, 338, 643-647.

(2) Kim, H. S.; Lee, C. R.; Im, J. H.; Lee, K. B.; Moehl, T.; Marchioro, A.; Moon, S. J.; Humphry-Baker, R.; Yum, J. H.; Moser, J. 
E.; et al. Lead Iodide Perovskite Sensitized All-Solid-State Submicron Thin Film Mesoscopic Solar Cell with Efficiency Exceeding 9\%. Sci. Rep. 2012, 2, No. 591.

(3) Yang, W. S.; Noh, J. H.; Jeon, N. J.; Kim, Y. C.; Ryu, S.; Seo, J.; Seok, S. I. High-Performance Photovoltaic Perovskite Layers Fabricated through Intramolecular Exchange. Science 2015, 348, 1234-1237.

(4) Burschka, J.; Pellet, N.; Moon, S. J.; Humphry-Baker, R.; Gao, P.; Nazeeruddin, M. K.; Gratzel, M. Sequential Deposition as a Route to High-Performance Perovskite-Sensitized Solar Cells. Nature 2013, 499, 316-319.

(5) Fang, Y.; Dong, Q.; Shao, Y.; Yuan, Y.; Huang, J. Highly Narrowband Perovskite Single-Crystal Photodetectors Enabled by Surface-Charge Recombination. Nat. Photonics 2015, 9, 679-686.

(6) Hao, F.; Stoumpos, C. C.; Cao, D. H.; Chang, R. P. H.; Kanatzidis, M. G. Lead-Free Solid-State Organic-Inorganic Halide Perovskite Solar Cells. Nat. Photonics 2014, 8, 489-494.

(7) Stranks, S. D.; Eperon, G. E.; Grancini, G.; Menelaou, C.; Alcocer, M. J.; Leijtens, T.; Herz, L. M.; Petrozza, A.; Snaith, H. J. Electron-Hole Diffusion Lengths Exceeding 1 Micrometer in an Organometal Trihalide Perovskite Absorber. Science 2013, 342, 341344.

(8) Dong, Q.; Fang, Y.; Shao, Y.; Mulligan, P.; Qiu, J.; Cao, L.; Huang, J. Electron-Hole Diffusion Lengths $>175 \mu \mathrm{m}$ in SolutionGrown $\mathrm{CH}_{3} \mathrm{NH}_{3} \mathrm{PbI}_{3}$ Single Crystals. Science 2015, 347, 967-970.

(9) Kim, J.; Lee, S. H.; Lee, J. H.; Hong, K. H. The Role of Intrinsic Defects in Methylammonium Lead Iodide Perovskite. J. Phys. Chem. Lett. 2014, 5, 1312-1317.

(10) Saidaminov, M. I.; Abdelhady, A. L.; Murali, B.; Alarousu, E.; Burlakov, V. M.; Peng, W.; Dursun, I.; Wang, L.; He, Y.; Maculan, G.; et al. High-Quality Bulk Hybrid Perovskite Single Crystals within Minutes by Inverse Temperature Crystallization. Nat. Commun. 2015, 6, No. 7586.

(11) Wu, X.; Trinh, M. T.; Niesner, D.; Zhu, H.; Norman, Z.; Owen, J. S.; Yaffe, O.; Kudisch, B. J.; Zhu, X. Y. Trap States in Lead Iodide Perovskites. J. Am. Chem. Soc. 2015, 137, 2089-2096.

(12) Niesner, D.; Zhu, H.; Miyata, K.; Joshi, P. P.; Evans, T. J.; Kudisch, B. J.; Trinh, M. T.; Marks, M.; Zhu, X. Y. Persistent Energetic Electrons in Methylammonium Lead Iodide Perovskite Thin Films. J. Am. Chem. Soc. 2016, 138, 15717-15726.

(13) Yang, Y.; Ostrowski, D. P.; France, R. M.; Zhu, K.; van de Lagemaat, J.; Luther, J. M.; Beard, M. C. Observation of a Hot-Phonon Bottleneck in Lead-Iodide Perovskites. Nat. Photonics 2016, 10, 5359.

(14) Savenije, T. J.; Ponseca, C. S., Jr.; Kunneman, L.; Abdellah, M.; Zheng, K.; Tian, Y.; Zhu, Q.; Canton, S. E.; Scheblykin, I. G.; Pullerits, T.; et al. Thermally Activated Exciton Dissociation and Recombination Control the Carrier Dynamics in Organometal Halide Perovskite. J. Phys. Chem. Lett. 2014, 5, 2189-2194.

(15) Guo, Z.; Wan, Y.; Yang, M.; Snaider, J.; Zhu, K.; Huang, L. Long-Range Hot-Carrier Transport in Hybrid Perovskites Visualized by Ultrafast Microscopy. Science 2017, 356, 59-62.

(16) Nie, W.; Tsai, H.; Asadpour, R.; Blancon, J. C.; Neukirch, A. J.; Gupta, G.; Crochet, J. J.; Chhowalla, M.; Tretiak, S.; Alam, M. A.; et al. High-Efficiency Solution-Processed Perovskite Solar Cells with Millimeter-Scale Grains. Science 2015, 347, 522-525.

(17) Reid, O. G.; Yang, M.; Kopidakis, N.; Zhu, K.; Rumbles, G. Grain-Size-Limited Mobility in Methylammonium Lead Iodide Perovskite Thin Films. ACS Energy Lett. 2016, 1, 561-565.

(18) Xing, G.; Mathews, N.; Sun, S.; Lim, S. S.; Lam, Y. M.; Gratzel, M.; Mhaisalkar, S.; Sum, T. C. Long-Range Balanced Electron- and Hole-Transport Lengths in Organic-Inorganic $\mathrm{CH}_{3} \mathrm{NH}_{3} \mathrm{PbI}_{3}$. Science 2013, 342, 344-347.

(19) Guo, Z.; Manser, J. S.; Wan, Y.; Kamat, P. V.; Huang, L. Spatial and Temporal Imaging of Long-Range Charge Transport in Perovskite Thin Films by Ultrafast Microscopy. Nat. Commun. 2015, 6, No. 7471.

(20) Tian, W.; Zhao, C.; Leng, J.; Cui, R.; Jin, S. Visualizing Carrier Diffusion in Individual Single-Crystal Organolead Halide Perovskite
Nanowires and Nanoplates. J. Am. Chem. Soc. 2015, 137, 1245812461.

(21) Hill, A. H.; Smyser, K. E.; Kennedy, C. L.; Massaro, E. S.; Grumstrup, E. M. Screened Charge Carrier Transport in Methylammonium Lead Iodide Perovskite Thin Films. J. Phys. Chem. Lett. 2017, 8, 948-953.

(22) Zigler, D. F.; Morseth, Z. A.; Wang, L.; Ashford, D. L.; Brennaman, M. K.; Grumstrup, E. M.; Brigham, E. C.; Gish, M. K.; Dillon, R. J.; Alibabaei, L.; et al. Disentangling the Physical Processes Responsible for the Kinetic Complexity in Interfacial Electron Transfer of Excited $\mathrm{Ru}(\mathrm{II})$ Polypyridyl Dyes on $\mathrm{TiO}_{2}$. J. Am. Chem. Soc. 2016, $138,4426-4438$.

(23) Wong, C. Y.; Penwell, S. B.; Cotts, B. L.; Noriega, R; Wu, H.; Ginsberg, N. S. Revealing Exciton Dynamics in a Small-Molecule Organic Semiconducting Film with Subdomain Transient Absorption Microscopy. J. Phys. Chem. C 2013, 117, 22111-22122.

(24) Gabriel, M. M.; Grumstrup, E. M.; Kirschbrown, J. R.; Pinion, C. W.; Christesen, J. D.; Zigler, D. F.; Cating, E. E.; Cahoon, J. F.; Papanikolas, J. M. Imaging Charge Separation and Carrier Recombination in Nanowire P-I-N Junctions Using Ultrafast Microscopy. Nano Lett. 2014, 14, 3079-3087.

(25) Johns, P.; Yu, K.; Devadas, M. S.; Hartland, G. V. Role of Resonances in the Transmission of Surface Plasmon Polaritons between Nanostructures. ACS Nano 2016, 10, 3375-3381.

(26) Yuan, L.; Wang, T.; Zhu, T.; Zhou, M.; Huang, L. Exciton Dynamics, Transport, and Annihilation in Atomically Thin TwoDimensional Semiconductors. J. Phys. Chem. Lett. 2017, 8, 3371-3379.

(27) Nah, S.; Spokoyny, B.; Stoumpos, C.; Soe, C. M. M.; Kanatzidis, M.; Harel, E. Spatially Segregated Free-Carrier and Exciton Populations in Individual Lead Halide Perovskite Grains. Nat. Photonics 2017, 11, 285-288.

(28) Baiz, C. R.; Schach, D.; Tokmakoff, A. Ultrafast 2D IR Microscopy. Opt. Express 2014, 22, 18724-18735.

(29) Ostrander, J. S.; Serrano, A. L.; Ghosh, A.; Zanni, M. T. Spatially Resolved Two-Dimensional Infrared Spectroscopy Via Wide-Field Microscopy. ACS Photonics 2016, 3, 1315-1323.

(30) Bi, D.; Moon, S.-J.; Häggman, L.; Boschloo, G.; Yang, L.; Johansson, E. M. J.; Nazeeruddin, M. K.; Grätzel, M.; Hagfeldt, A. Using a Two-Step Deposition Technique to Prepare Perovskite $\left(\mathrm{CH}_{3} \mathrm{NH}_{3} \mathrm{PbI}_{3}\right)$ for Thin Film Solar Cells Based on $\mathrm{ZrO}_{2}$ and $\mathrm{TiO}_{2}$ Mesostructures. RSC Adv. 2013, 3, 18762-18766.

(31) Bao, C.; Chen, Z.; Fang, Y.; Wei, H.; Deng, Y.; Xiao, X.; Li, L.; Huang, J. Low-Noise and Large-Linear-Dynamic-Range Photodetectors Based on Hybrid-Perovskite Thin-Single-Crystals. Adv. Mater. 2017, 29, No. 1703209.

(32) Polin, M.; Ladavac, K.; Lee, S.-H.; Roichman, Y.; Grier, D. G. Optimized Holographic Optical Traps. Opt. Express 2005, 13, 58315845.

(33) Lohmann, A. W.; Paris, D. P. Binary Fraunhofer Holograms, Generated by Computer. Appl. Opt. 1967, 6, 1739-1748.

(34) Stranks, S. D.; Burlakov, V. M.; Leijtens, T.; Ball, J. M.; Goriely, A.; Snaith, H. J. Recombination Kinetics in Organic-Inorganic Perovskites: Excitons, Free Charge, and Subgap States. Phys. Rev. Appl. 2014, 2, No. 034007.

(35) Manser, J. S.; Kamat, P. V. Band Filling with Free Charge Carriers in Organometal Halide Perovskites. Nat. Photonics 2014, 8, 737-743.

(36) Yamada, Y.; Nakamura, T.; Endo, M.; Wakamiya, A.; Kanemitsu, Y. Photocarrier Recombination Dynamics in Perovskite $\mathrm{CH}_{3} \mathrm{NH}_{3} \mathrm{PbI}_{3}$ for Solar Cell Applications. J. Am. Chem. Soc. 2014, 136, 1161011613.

(37) Yamada, Y.; Yamada, T.; Phuong le, Q.; Maruyama, N.; Nishimura, H.; Wakamiya, A.; Murata, Y.; Kanemitsu, Y. Dynamic Optical Properties of $\mathrm{CH}_{3} \mathrm{NH}_{3} \mathrm{PbI}_{3}$ Single Crystals as Revealed by One- and Two-Photon Excited Photoluminescence Measurements. J. Am. Chem. Soc. 2015, 137, 10456-10459.

(38) Jain, S. C.; McGregor, J. M.; Roulston, D. J. Band-Gap Narrowing in Novel III-V Semiconductors. J. Appl. Phys. 1990, 68, $3747-3749$ 
(39) Grancini, G.; Srimath Kandada, A. R.; Frost, J. M.; Barker, A. J.;

De Bastiani, M.; Gandini, M.; Marras, S.; Lanzani, G.; Walsh, A.;

Petrozza, A. Role of Microstructure in the Electron-Hole Interaction of

Hybrid Lead-Halide Perovskites. Nat. Photonics 2015, 9, 695-701. 